\title{
Optimal scheduling of a household refrigerator using adaptive model predictive technique
}

\author{
Roland Bálint \\ and Information Systems \\ Faculty of Information Technology \\ University of Pannonia
}

Egyetem street 10, Veszprém, Hungary

Email: balint.roland@ virt.uni-pannon.hu

\author{
Attila Magyar \\ Department of Electrical Engineering \\ and Information Systems \\ Faculty of Information Technology \\ University of Pannonia
}

Egyetem street 10, Veszprém, Hungary

Email: magyar.attila@virt.uni-pannon.hu

\begin{abstract}
A heuristic and adaptive model predictive control algorithm is presented in this work which operates domestic refrigerators connected to an electrical grid operating in a dayahead market (DAM) and temperature constraints also have to be met by the refrigerator temperatures.

The problem has been solved using the model predictive framework using a simple refrigerator model. This paper is a continuation of a previous work that has been extended so that the optimal scheduler algorithm adapts its steps to the actual estimated interior heat capacity of the refrigerator (which depends on the food quantity, food temperature, etc. in the interior).

It is shown that the proposed adaptive MPC based scheduling algorithm gives a better performance (with respect to price) as opposed to the scheduling algorithm having no precise knowledge of the actual load of the refrigerator. Moreover, due to the adaptive behavior it was possible to decrease the running time of the optimal scheduler algorithm compared to the non-adaptive version.
\end{abstract}

\section{INTRODUCTION}

One of the most important elements in living on the Planet in a sustainable way is to manage the energy consumed by the domestic sector efficiently. In order to meet the challenges of sustainable energy consumption, a huge number of developments are being made under the flag of smart grid technologies. An important element of these solutions is the so-called day-ahead electricity market (DAM), that is continuously expanding, and the amount of energy being traded through them is increasing. As a result of optimized pricing, hourly changing electrical energy prices are available for the day-ahead electricity market, see Fig. 1.

In spite of the continuous development of control methods available for solving optimal control problems, they did not infiltrate the domestic application area, where usually bangbang or PID type controllers have been used. Any usage of computationally demanding algorithms in such applications was prohibitive. However, in the past few years (or decade) low power micro-controllers (MCU) with high computational potential have revolutionized the field of embedded systems thus opening the way to apply advanced control methods in this area, too. In [2] a mixed integer linear programming based approach is used for the optimal scheduling of domestic

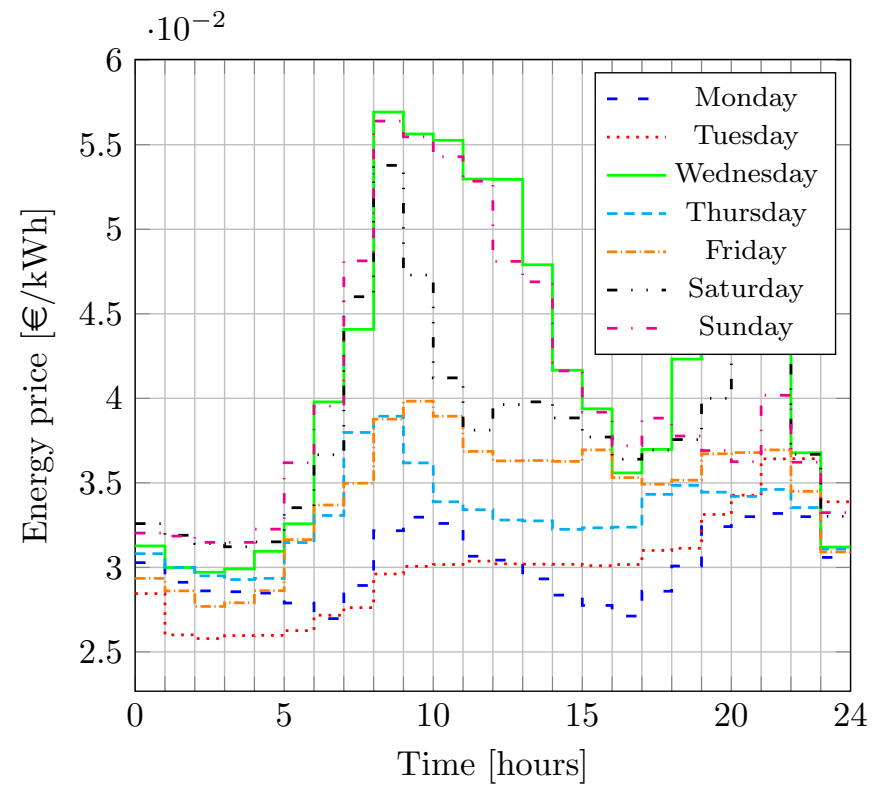

Fig. 1. Price signals for the days of a week in a day-ahead market. Source: [1]

appliancess in a smart environment. On the other hand, cooperative particle swarm optimization and similar soft computing methods (e.g. [3]) are also applicable fo energy consumption optimization in smart home applications.

Model predictive control (MPC) [4] is widely celebrated both in the industrial and in the academic areas because of its massive theoretical background and the capability to tackle multivariable control problems at the same time [5]. In a control application using MPC as its control scheme, a reliable dynamical model of the controlled dynamical system is needed. The authors of the work [6] propose a grey-box modelling approach for household refrigerators as a basis of a demand side management (DSM) application. An optimal scheduling algorithm has been given in [7] where the optimal scheduling of different household appliances has been formulated as a nonlinear integer programming problem and solved 
by genetic algorithm.

Model based control developments enable the engineers to perform simulation experiments in the control design phase since the dynamical model of the system to be controlled is known. In the work [8], a simulation software tool is proposed for the optimal scheduling of smart power appliances.

The online estimation of the model parameters (e.g. in a varying temperature situation) enables the controller to adjust the used model to the actual system, e.g. in the work [9] the demand response potential of a refrigerator system being used in a supermarket has been proposed together with an estimator of the actual food temperature.

The starting point for the current work is the development presented by [10] where MPT toolbox [11] available in Matlab has been used for the cost optimal operation of household refrigerators [12] in the constant parameter case. The optimizer algorithm behind the model predictive scheduler has been changed to an adaptive branch-and-bound binary search algorithm capable of estimating the actual load (food) of the refrigerator. The aim of our current work is to be able to estimate the critical model parameter of the system on-line, and thus making our method adaptive.

\section{Piece-Wise AfFine Model of the Refrigerator}

\section{A. System description}

Household refrigerators consists of two containments, one being their container and another one being their wall as it is shown in Fig. 2. The walls towards the outer environment can be assumed perfectly isolating, while the inner container is usually cooled by a cooling liquid circuit driven by an electrical motor through the back wall. The refrigerator can

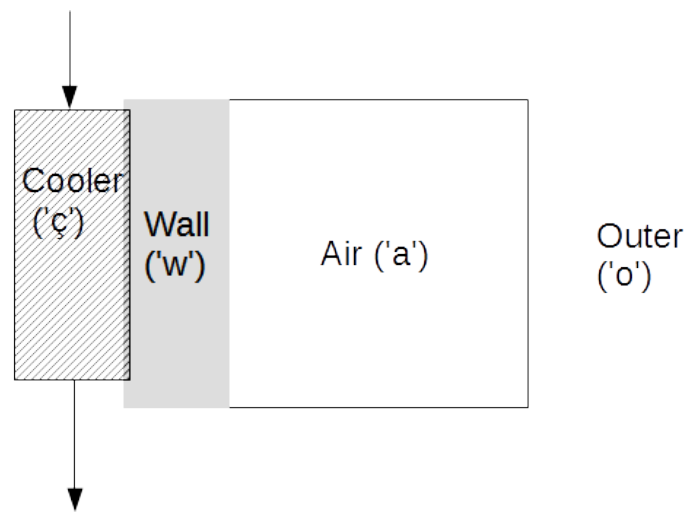

Fig. 2. The schematic picture of the refrigerator

work in two operating modes: the cooling and the reheating modes. An "on/off" type switch $S$ changes the operating modes. The inner container's temperature $T_{a}$ has a minimum $T_{a, \min }$ and a maximum $T_{a, \max }$ temperature to be respected by the controller.

\section{B. Piecewise affine model}

A simple dynamic model of the refrigerator was developed in [12] using first engineering principles, i.e. energy conser- vation for the refrigerator container (denoted by a subscript ${ }_{a}$ ) and for its wall (denoted by a subscript ${ }_{w}$ ). Thus the state variables of the model are the container and the wall temperatures ( $T_{a}$ and $T_{w}$ ), respectively, and the input variable is the status of the switch $S$.

In both operating modes (cooling and reheating) the state space model $\Sigma$ is in the following form

$$
\begin{aligned}
& \dot{\boldsymbol{x}}=\boldsymbol{A} \boldsymbol{x}+\boldsymbol{B} \boldsymbol{u} \\
& \boldsymbol{y}=\boldsymbol{C} \boldsymbol{x}
\end{aligned}
$$

but the value of the coefficient matrices $A, B, C$ differ. The input- and state variables are

$$
\boldsymbol{x}=\left[\begin{array}{ll}
T_{a} & T_{w}
\end{array}\right]^{T}, \quad \boldsymbol{u}=\left[\begin{array}{ll}
S & T_{o}
\end{array}\right]^{T} .
$$

Cooling dynamics $\left(\Sigma_{o n}\right)$ : The first case is when the switch is closed $(S=1)$, i.e. the refrigerator is cooling. Then the parameter matrices are

$$
\begin{aligned}
& \boldsymbol{A}_{\text {on }}=\left[\begin{array}{cc}
-\frac{K_{w}+K_{o}}{C_{a}} & \frac{K_{w}}{C_{a}} \\
\frac{K_{w}}{C_{w}} & -\left(\frac{K_{w}}{C_{w}}+\frac{K_{c}}{C_{w}}+\frac{K_{x}}{C_{w}}\right)
\end{array}\right] \\
& \boldsymbol{B}_{\text {on }}=\left[\begin{array}{cc}
0 & \frac{K_{o}}{C_{a}} \\
\frac{T_{c} K_{c}}{C_{w}} & \frac{K_{x}}{C_{w}}
\end{array}\right], \boldsymbol{C}=\left[\begin{array}{ll}
1 & 0 \\
0 & 1
\end{array}\right] .
\end{aligned}
$$

The physical meaning and the estimated values of the parameters as reported in our previous paper [12] can be found in Table I. It is important to note that the heat capacity of the containment $C_{a}$ varies with the varying load of the refrigerator, while all the other parameters can be assumed to be constant.

Reheating dynamics $\left(\Sigma_{o f f}\right)$ : The second case is when the switch is open $(S=0)$, i.e. the refrigerator is reheated to the environmental temperature. Then the parameter matrices are as follows:

$$
\begin{gathered}
\boldsymbol{A}_{\text {off }}=\left[\begin{array}{cc}
-\frac{K_{w}+K_{o}}{C_{a}} & \frac{K_{w}}{C_{a}} \\
\frac{K_{w}}{C_{w}} & -\left(\frac{K_{w}}{C_{w}}+\frac{K_{x}}{C_{w}}\right)
\end{array}\right] \\
\boldsymbol{B}_{\text {off }}=\left[\begin{array}{ll}
0 & \frac{K_{o}}{C_{a}} \\
0 & \frac{K_{x}}{C_{w}}
\end{array}\right], \boldsymbol{C}=\left[\begin{array}{ll}
1 & 0 \\
0 & 1
\end{array}\right] .
\end{gathered}
$$

\section{AdAptive Model Predictive Control}

\section{A. Control aim}

The controller to be designed has to meet the following control specifications.

- The control input is the state of the switch that controls the operation mode, i.e. it is a binary value (off: $S=0$, on: $S=1)$.

- The outer (environmental) temperature input of the system is supposed to be constant ( $T_{o}=$ constant).

- The price of the consumed electrical energy is the operating cost.

- The value of the electrical energy price $p(t)$ changes hourly during the day and is known for next 24 hours in advance.

- The operating temperature constraints of the refrigerator are the following: 
- $T_{a, \min }$ and $T_{a, \max }$ are the minimal and maximal inner air temperatures $\left(T_{a}\right)$,

- $T_{w, \min }$ and $T_{w, \max }$ are the minimal and maximal back wall temperatures $\left(T_{w}\right)$.

- The inner heat capacity changes between the heat capacity of the empty refrigerator $\left(C_{a_{m i n}}\right)$ and a maximal value $\left(20 C_{a_{m i n}}\right)$.

- The rate of change of the interior heat capacity is limited $\left(\left|\Delta C_{a}\right|<5 C_{a_{m i n}}\right)$.

- If the termperature difference between the model and the real system exceeds a predefined error limit $(\Delta T)$, then the interior heat capacity $C_{a}$ has to be re-estimated by the controller.

- The controller has to be optimal with respect to the operating cost, defined by the formula (7):

$$
\int_{\tau=0}^{24 h}(p(\tau) \cdot S(\tau)) d \tau \text {. }
$$

\section{B. Model Predictive Scheduling}

The heuristic model predictive control algorithm proposed in [12] meets almost all the above control aims except for the adaptivity with respect to the changes in the interior heat capacity caused by the quantity of food occassionally put in and removed from the refrigerator. It also works if $C_{a}$ is unknown (i.e. the refrigerator is supposed to be empty), however the scheduled operation is not optimal with respect to (7).

The model predictive scheduler described in [12] serves as a starting point of the present work and its operation is summarized in what follows. The optimization algorithm behind the MPC problem to be solved in each iteration is a heuristic branch and bound binary search algorithm based on the following three rules:

Rule 1: Any scheduling sequence that yields a state variable $\boldsymbol{x}$ breaking the temperature bounds is not allowed.

Rule 2: Any scheduling sequence that yields a higher $\boldsymbol{x}$ at a higher cost is not optimal.

Rule 3: Any scheduling sequence containing a cooling step that could have been performed later for a lower energy price is not optimal.

In order to assure that the model predictive scheduler works properly the actual value of $C_{a}$ should be determined online and the parameter matrices of both dynamics must be modified accordingly.

\section{Adaptive Model Predictive Scheduling}

The adaptivity of the model predictive scheduler is implemented by a parameter estimation step in which the actual value of the refrigerator interior heat capacity is being determined based on the available measurements. As a result, a possibly different value of the parameter $C_{a}=C_{a}(n)$ is obtained in each iteration $n$ of the model predictive scheduler. The pseudocode of the adaptive extension of the proposed algorithm is listed in Algorithm 1, where $\Sigma_{o n}$ and $\Sigma_{o f f}$ denotes the discretized version of the two dynamics (3)-(4) and

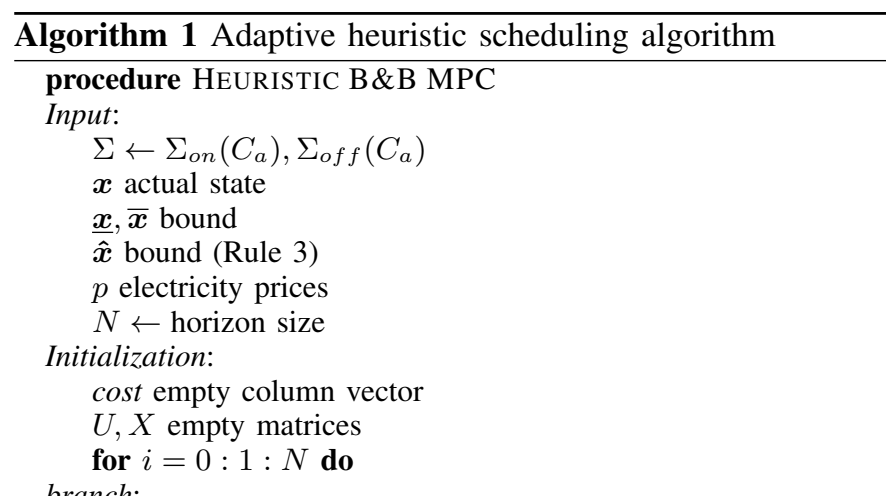
branch:

$$
\begin{aligned}
& U=\left[\begin{array}{c|c}
U & 1 \\
& \vdots \\
& 1 \\
U & 0 \\
& \vdots \\
0
\end{array}\right], X=\left[\begin{array}{l}
X \\
\hline X
\end{array}\right], \text { cost }=\left[\begin{array}{c}
\text { cost } \\
\text { cost }
\end{array}\right] \\
& \text { for } k=1: \operatorname{rows}(U) \text { do } \\
& \quad X_{k, i+1}=\Sigma\left(X_{k, i}, U_{k, i}\right) \\
& \quad \text { update } \operatorname{cost} t_{k}
\end{aligned}
$$

bound (Rule 1):

if $X_{k, i+1} \notin[\underline{x}, \bar{x}]$ then end if

delete row $X_{k, .}, U_{k, .}$ and $\operatorname{cost}_{k}$

bound (Rule 3):

$$
\text { if } X_{k, i+1}<\hat{\boldsymbol{x}}_{k} \text { and } U_{k, i+1}=1 \text { then }
$$$$
\text { delete row } X_{k, .}, U_{k, .} \text { and } \text { cost }_{k}
$$
end if

end for

bound (Rule 2):

for $k, l=1: \operatorname{rows}(U), k \neq l$ do

if $X_{k, i+1}>X_{l, i+1}$ and $\operatorname{cost}_{k}>$ cost $_{l}$ then

else delete row $X_{k, .}, U_{k, .}$, cost $_{k}$

if $X_{l, i+1}>X_{k, i+1}$ and cost $_{l}>$ cost $_{k}$ then end if delete row $X_{l, .}, U_{l, .}$, cost $_{l}$

end if

end for

end for

optimal solution:

minimal value of $\operatorname{cost}=\operatorname{cost}_{k_{o p t}}$

Minimizing sequence $U_{k_{o p t}}$.

end procedure

procedure ADAPTIVE SCHEDULING METHOD

Input:

$h$ sampling time

$P=\left[T c, K w, K o, K c, C a, C w, K x, C a_{m i n}\right]$ system parameters

Scheduling method:

for (in each $h$ time step) do

measure the temperatures of the refrigerator $X_{m e s}$ if $X_{m e s}-X_{s i m}>\Delta T$ then

end if

RUN $C_{a}$ estimation

RUN Heuristic B\&B MPC Procedure with $C_{a}$

end for

end procedure 
(5)-(6), respectively. The novel element is the online parameter estimation performed in the begining of the control loop. The online estimation of the interior heat capacity is based on the previous temperature measurements.

The online estimation of $C_{a}$ : is performed at the beginning of a control cycle if the difference between the model output temperature and the measured temperature is greater than the predefined $\Delta T$ value. The estimation is basically a bisection method used for finding the root of the difference $\Delta T_{a}\left(C_{a}\right)$ (8) with respect to the heat capacity parameter $C_{a}$.

$$
\Delta T_{a}\left(C_{a}\right)=\hat{T}_{a}(n)-T_{a}\left(C_{a}, n\right)
$$

where $\hat{T}_{a}(n)$ is the measured interior air temperature at the discrete time instant $n$, and $T_{a}\left(C_{a}, n\right)$ is the interior air temperature computed from the discretized version of model (3)-(4) or (5)-(6), depending on the state of the switching input $U(n)$. During the search, the parameter $C_{a}$ is tuned iteratively in the parametric (i.e. $C_{a}$ dependent) discrete-time state space model parameter matrices (9-10) of the corresponding operation mode. The discrete-time parametric state space model matrices are

$$
\begin{aligned}
& \boldsymbol{\Phi}\left(C_{a}\right)= e^{\boldsymbol{A}\left(\boldsymbol{C}_{\boldsymbol{a}}\right) \cdot \boldsymbol{t}_{\boldsymbol{s}}}= \\
&= {\left[\begin{array}{c}
\frac{-s_{1}+a_{22}}{s_{2}-s_{1}} e^{s_{1} h}+\frac{s_{2}-a_{22}}{s_{2}-s_{1}} e^{s_{2} h} \\
\frac{-a_{21}}{\left(s_{2}-s_{1}\right)} e^{s_{1} h}+\frac{a_{21}}{\left(s_{2}-s_{1}\right)} e^{s_{2} h} \\
\frac{-a_{12}}{\left(s_{2}-s_{1}\right) C_{a}} e^{s_{1} h}+\frac{a_{12}}{\left(s_{2}-s_{1}\right) C_{a}} e^{s_{2} h} \\
\frac{-s_{1}+a_{11}}{\left(s_{2}-s_{1}\right) C_{a}} e^{s_{1} h}+\frac{s_{2}-a_{11}}{\left(s_{2}-s_{1}\right) C_{a}} e^{s_{2} h}
\end{array}\right] } \\
& \boldsymbol{\Gamma}\left(C_{a}\right)=\boldsymbol{A}\left(\boldsymbol{C}_{\boldsymbol{a}}\right)^{-\mathbf{1}}\left(\boldsymbol{\Phi}\left(\boldsymbol{C}_{\boldsymbol{a}}\right)-\boldsymbol{I}\right) \boldsymbol{B}\left(\boldsymbol{C}_{\boldsymbol{a}}\right),
\end{aligned}
$$

where

$$
s_{1,2}=\frac{a_{11}+C_{a} a_{22} \pm \sqrt{\left(a_{11}+C_{a} a_{22}\right)^{2}-4 C_{a}\left(a_{11} a_{22}-a_{12} a_{21}\right)}}{2 C_{a}},
$$

and

$$
\begin{gathered}
\boldsymbol{A}\left(C_{a}\right)=\left[\begin{array}{ll}
\frac{a_{11}}{C_{a}} & \frac{a_{12}}{C_{a}} \\
a_{21} & a_{22}
\end{array}\right] \\
\boldsymbol{B}\left(C_{a}\right)=\left[\begin{array}{ll}
\frac{b_{11}}{C_{a}} & \frac{b_{12}}{C_{a}} \\
b_{21} & b_{22}
\end{array}\right] .
\end{gathered}
$$

The search interval for the value of $C_{a}(n)$ is an interval of length $10 C_{a_{\min }}$ with one of the end points being the previous guess for the interior heat capacity, i.e. $C_{a}(n-1)$. The number of necessary iterations of the above bisection algorithm depends on the desired tolerance $\varepsilon$ in the following form

$$
N_{C_{a}}=\log _{2}\left(\frac{10 C_{a_{m i n}}}{\varepsilon C_{a_{\text {min }}}}\right)=\log _{2}\left(\frac{10}{\varepsilon}\right) .
$$

\section{Simulation EXPERIMENTS}

The simulation experiments have been performed using the parameter values listed in Table I.
TABLE I

PARAMETER VALUES OF THE CASE STUDY

\begin{tabular}{|l|c|c|c|}
\hline parameter & symbol & value & unit \\
\hline sampling time & $h$ & 300 & $\mathrm{~s}$ \\
outer air temperature & $T_{o}$ & 20 & ${ }^{\circ} \mathrm{C}$ \\
cooling liquid temperature & $T_{c}$ & -36.71 & ${ }^{\circ} \mathrm{C}$ \\
minimal inner air temperature & $T_{a, \min }$ & 0.1 & ${ }^{\circ} \mathrm{C}$ \\
maximal inner air temperature & $T_{a, \max }$ & 5.5 & ${ }^{\circ} \mathrm{C}$ \\
minimal back wall temperature & $T_{w, \min }$ & -19 & ${ }^{\circ} \mathrm{C}$ \\
maximal back wall temperature & $T_{w, \max }$ & 5.8 & ${ }^{\circ} \mathrm{C}$ \\
air-wall heat transfer coeff. & $K_{w}$ & $3.78 \cdot 10^{3}$ & $\frac{\mathrm{kW}}{\mathrm{o}} \mathrm{C}$ \\
air-env. heat transfer coeff. & $K_{o}$ & $2.04 \cdot 10^{3}$ & $\frac{\mathrm{kW}}{\mathrm{o}}$ \\
wall-env. heat transfer coeff. & $K_{x}$ & $0.52 \cdot 10^{3}$ & $\frac{\mathrm{kW}}{\mathrm{o}}$ \\
wall-cool. liq. heat transfer coeff. & $K_{c}$ & $5.01 \cdot 10^{3}$ & $\frac{\mathrm{kW}}{\circ} \mathrm{C}$ \\
heat capacity of empty containment & $C_{a_{\min }}$ & $1.21 \cdot 10^{7}$ & $\frac{\mathrm{kJ}}{\mathrm{o} \mathrm{C}}$ \\
heat capacity of wall & $C_{w}$ & $3.41 \cdot 10^{6}$ & $\frac{\mathrm{KJ}}{{ }^{\circ} \mathrm{C}}$ \\
\hline
\end{tabular}

\section{A. The execution time of the interior heat capacity estimation}

The model parameter estimation step involving the bisection search for the parameter $C_{a}$ has been implemented both by rediscretizing the continuous time dynamics and by evaluating the matrices (9-10). The former approach is supposed to be computationally more demanding since the matrix exponential function has to be computed in each iterations.

Table II summarizes the difference. Note, that the calculations are being performed in Matlab, using a middle class computer. As expected, the parametric discrete time model

TABLE II

EXECUTION TIME OF THE $C_{a}$ ESTIMATION USING BISECTION PROCEDURE

\begin{tabular}{c|c|c} 
& re-discretizing & parametric model \\
\hline running time $[\mathrm{ms}]$ & $37-40$ & $0.35-0.4$
\end{tabular}

performs far better than the other approach which makes it a perfect candidate to be used in a future MCU implementation of the scheduling algorithm. Note, that using $1 \%$ tolerance, the typical value of $N_{C_{a}}$ (13) was 6-7 bisection steps in the simulation using system parameters listed in Table I.

\section{B. The effect of the adaptivity on the heuristic MPC}

Heat capacity is among the most important parameters of thermal systems and it has a serious effect on the dynamics (e.g. time constant) of a thermal system. It is expected that the more precise knowledge of $C_{a}$ enables the adaptive heuristic scheduler to approach the optimal solution. Fig. 3 gives an overview on the effect of adaptivity on the scheduling.

The behavior of the non-adaptive model predictive scheduler [12] is shown on the top plot of the figure. It can be seen that the simulated (dotted line) and measured (solid line) temperatures are almost identical in the first and in the last hours since the actual interior heat capacity coincides with the value used by the model predictive scheduler in these intervals. Between 2 and 20 hours, however, the actual heat capacity changes, and the scheduler uses the $C_{a}$ value corresponding to the heat capacity of an empty refrigerator resulting in a suboptimal operation that still obeys the temperature constraints. The oscillations of the simulations as opposed 
to the measurements can be explained by the different time constants of the modelled (faster) and the actual (slower) thermal dynamics. The actual heat capacity of the system is plotted by solid line in the bottom plot of Fig. 3 .

The second plot of Fig. 3 shows the interior temperature $\left(T_{a}\right)$ for both the actual (solid line) and modelled dynamics (dotted line). It is apparent, that due to the smaller difference between the model output and the real measurements, it was possible for the scheduler to keep the actual $T_{a}$ on a higher level, but still within the constraints. This results in a lower energy consumption and price, due to the information about the actual heat capacity of the system. The threshold $\Delta T$ of the adaptive parameter estimation was $0.1^{\circ} \mathrm{C}$, i.e. if the difference between measured and modelled temperature is bigger than $0.1^{\circ} \mathrm{C}$, then the algorithm estimates the actual heat capacity and the MPC can calculates the next control input with the updated parameter. The estimated $C_{a}$ (dotted line) together with its actual value solid line can be seen on the bottom plot.

The third plot shows the same signals as the second one with the only difference being that the threshold temperature difference of the online parameter estimation algorithm is $0.01^{\circ} \mathrm{C}$.

Based on the experiments it can be stated that the $0.1^{\circ} \mathrm{C}$ (second plot) gives the adaptive heuristic model based scheduler enough information about the change of the interior heat capacity. The operating cost of using non-adaptive model predictive scheduler is $25 \%$ less than that of the traditional refrigerator control [10]. The performed simulation experiments also show that the operating cost of the refrigerator scheduled using the adaptive heuristic MPC algorithm is 3\% below the operating cost of the non-adaptive one. In an average household, $15-20 \%$ of the total electrical consumption corresponds to the operating of the refrigerator. With the proposed adaptive model predictive solution the total electrical consumption of the household decreases with $3-5 \%$. Moreover, according to Table III, it can be stated that the adaptive extension of the heuristic model predictive scheduler can be calculated faster than the non-adaptive scheduler [12]. The explanation of this phenomena is the value of the interior heat capacity can only be higher than the value of an empty refrigerator $\left(C_{a_{m i n}}\right)$ used by the non-adaptive algorithm. The adaptively identified dynamics of the scheduler gets slower due to the increased $C_{a}$ value which can be calculated using less input calculation (Algorithm 1).

TABLE III

THE CALCULATION TIMES OF NON-ADAPTIVE AND ADAPTIVE MPC FOR A WHOLE DAY

\begin{tabular}{c|c|c} 
& non-adaptive MPC & adaptive MPC \\
\hline running time [s] & $175-180$ & $45-55$
\end{tabular}

\section{CONCLUSiON}

The price optimal scheduling of domestic refrigerators connected to an electrical grid operating in a day-ahead market (DAM) has been investigated in this paper. The optimal scheduling problem has been solved using the model predictive framework using a simple piecewise affine refrigerator model.
A previously developed preliminary solution of the problem has been extended so that the optimal heuristic scheduler algorithms adapts its steps to the actual estimated interior heat capacity of the refrigerator (which depends on the food quantity, food temperature, etc. in the interior).

It is shown that the proposed adaptive MPC based scheduling algorithm gives a better performance (with respect to price) as opposed to the scheduling algorithm having no precise knowledge of the actual load of the refrigerator. Moreover, due to the adaptive behavior it was possible to decrease the running time of the optimal scheduler algorithm compared to the non-adaptive version of the algorithm which is crucial from the MCU implementation point of view.

The further work includes the verification of the proposed optimal adaptive scheduling algorithm using a measurement dataset obtained from an industrial refrigerator compartment.

\section{ACKNOWLEDGMENT}

This research is supported by the National Research, Development and Innovation Office - NKFIH through grant No.115694.

A. Magyar was supported by the János Bolyai Research Scholarship of the Hungarian Academy of Sciences.

\section{REFERENCES}

[1] E. Spot, "Market data," System price.[Verkkotietokanta][Viitattu 23.11. 2011] Saatavissa: http://www. nordpoolspot. com/Marketdata1/Elspot/Area-Prices/ALL1/Hourly/http://www. nordpoolspot. com/Market-datal/Elspot/Area-Prices/ALL1/Hourly, 2010.

[2] K. C. Sou, J. Weimer, H. Sandberg, and K. H. Johansson, "Scheduling smart home appliances using mixed integer linear programming," in 2011 50th IEEE Conference on Decision and Control and European Control Conference, Dec 2011, pp. 5144-5149.

[3] M. A. A. Pedrasa, T. D. Spooner, and I. F. MacGill, "Coordinated scheduling of residential distributed energy resources to optimize smart home energy services," IEEE Transactions on Smart Grid, vol. 1, no. 2 , pp. 134-143, Sept 2010.

[4] J. Ma, S. J. Qin, and T. Salsbury, "Application of economic mpc to the energy and demand minimization of a commercial building," Journal of Process Control, vol. 24, no. 8, pp. 1282-1291, 2014.

[5] E. Rodrigues, R. Godina, E. Pouresmaeil, J. Ferreira, and J. Catalão, "Domestic appliances energy optimization with model predictive control," Energy Conversion and Management, vol. 142, pp. 402-413, 2017.

[6] F. Sossan, V. Lakshmanan, G. T. Costanzo, M. Marinelli, P. J. Douglass, and H. Bindner, "Grey-box modelling of a household refrigeration unit using time series data in application to demand side management," Sustainable Energy, Grids and Networks, vol. 5, pp. 1-12, 2016.

[7] D. Setlhaolo and X. Xia, "Optimal scheduling of household appliances incorporating appliance coordination," Energy Procedia, vol. 61, pp. 198-202, 2014

[8] V. Kaczmarczyk, P. Fiedler, Z. Bradac, L. Franek, and J. Pasek, "Simulator for optimal scheduling of domestic appliances," IFAC-PapersOnLine, vol. 48, no. 4, pp. 95-100, 2015.

[9] R. Pedersen, J. Schwensen, B. Biegel, T. Green, and J. Stoustrup, "Improving demand response potential of a supermarket refrigeration system: A food temperature estimation approach," IEEE Transactions on Control Systems Technology, vol. 25, no. 3, pp. 855-863, 2017.

[10] R. Bálint and A. Magyar, "Refrigerator optimal scheduling to minimize the cost of operation," Hungarian Journal of Industriy and Chemistry, vol. 44, no. 2, pp. 99-104, 2016.

[11] M. Herceg, M. Kvasnica, C. Jones, and M. Morari, "Multi-parametric toolbox 3.0," in Proceedings of the European Control Conference, no. EPFL-CONF-186265, 2013.

[12] R. Bálint, A. Magyar, and K. M. Hangos, "Cost-optimal model predictive scheduling of home appliances," in Proceedings of the 20th IFAC World Congress, Toulouse, France. IFAC, 2017, p. accepted. 


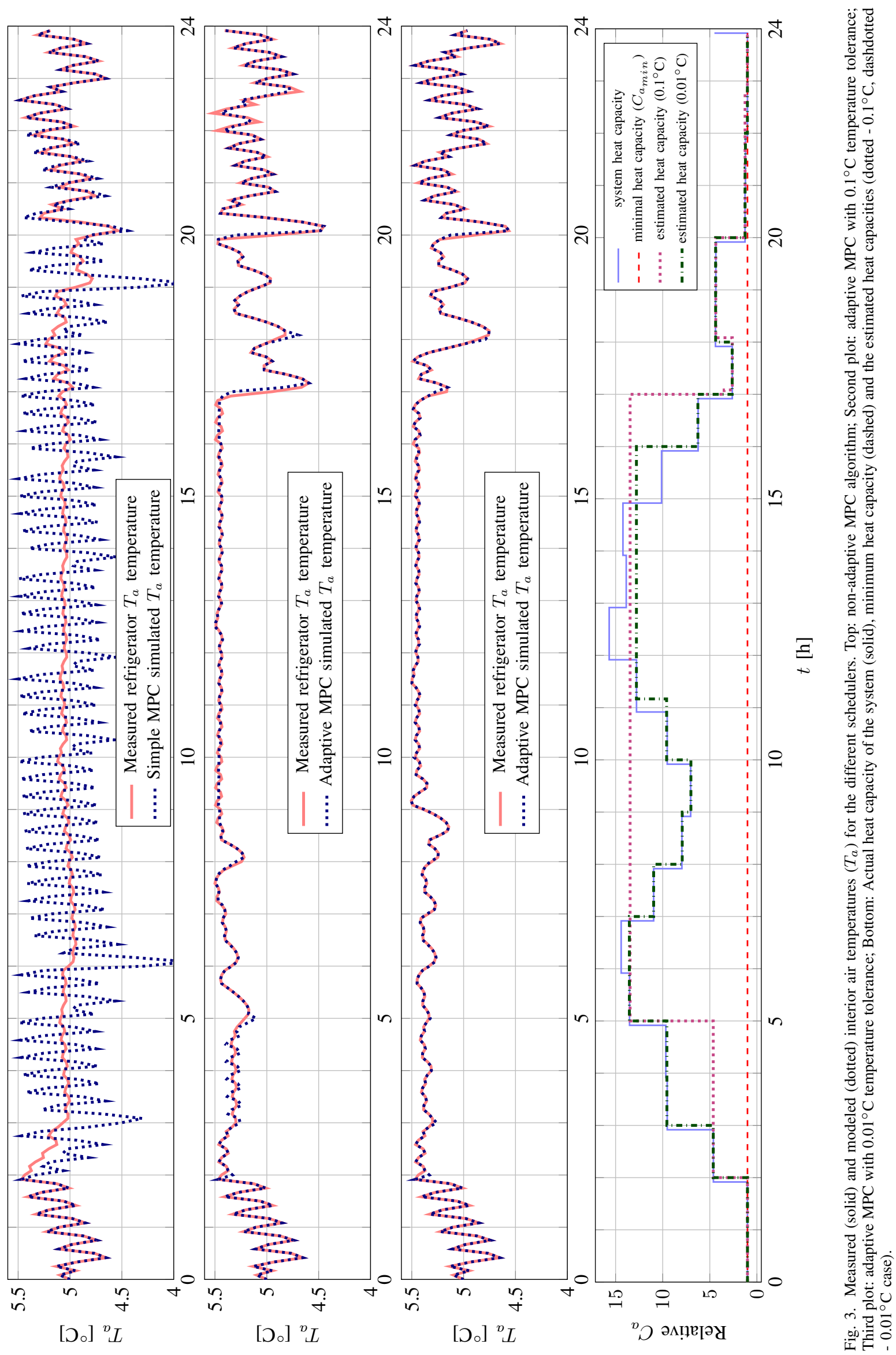

